\title{
Collaborative and Cooperative Environments
}

\author{
Christoph Anthes ${ }^{1}$, Vassil Alexandrov ${ }^{2}$, Dieter Kranzlmüller ${ }^{1}$, \\ Jens Volkert ${ }^{1}$, and Gerhard Widmer ${ }^{3}$ \\ ${ }^{1}$ GUP, Institute of Graphics and Parallel Processing \\ Johannes Kepler University, Altenbergerstraße 69, A-4040 Linz, Austria \\ ${ }^{2}$ Centre for Advanced Computing and Emerging Technologies, \\ The University of Reading, Reading, RG6 6AY, United Kingdom \\ ${ }^{3} \mathrm{CP}$, Institute of Computational Perception \\ Johannes Kepler University, Altenbergerstraße 69, A-4040 Linz, Austria
}

\section{Motivation}

Technological advances in high-speed networking and computational grids do not only transform the methods applied to everyday science, but also the collaboration and cooperation between scientists at almost arbitrary locations around the world. The additional provision of multi-sensory, immersive Virtual Reality interfaces as tools to improve the collaboration between groups of human users is another hot topic in this research domain, which will most likely increase the potential benefits of these distributed research communities. The vision to facilitate large scale, complex simulations, which may be steered through natural and intuitive interfaces, is both intriguing and of high scientific interest.

This workshop at the ICCS 2008 in Cracow comprises the best submitted papers concerning the application and usage of collaborative and cooperative environments, as well as the technologies supporting them in the scientific and industrial context. The workshop on collaborative and cooperative environments has offered the possibility to discuss the different approaches in this domain, to show the latest results, products, or research prototypes to potential users, and to establish connections between developers and users of associated technologies. The attendants were asked to present and discuss the following technologies:

- Collaborative and cooperative tools and environments

- Development of associated parallel and distributed computing solutions

- Integration of networking and grid computing technology

- Provision of multi-sensory, natural and intuitive interfaces

- Immersive Virtual and Augmented Reality approaches

- Requirement studies for future collaboration tools

- Evaluation of existing collaboration environments and practical experiences

Each of the submitted papers has been reviewed by at least three international referees in this domain. The highest ranking contributions are presented here. 


\section{Overview of Contributions}

The paper by Choiński et al., Multi-Agent System for Collaboration in Hybrid Control, investigates an ontology-based multi-agent system augmented with web engineering for the for validation of hybrid control of biotechnological plant. In order to acquire knowledge and configure the multi-agent system, the data gathered from the web is reduced, filtered and validated.

The problem of geographically distributed software engineering is addressed by Penichet et al. in their contribution Design and Evaluation of a Service Oriented Architecture-based Application to Support the Collaborative Edition of UML Class Diagrams. The authors present a collaborative CASE tool called CE4WEB to support the edition of UML diagrams using the Service Oriented Architecture paradigm.

Another contribution from the domain of software engineering, g-Eclipse a contextualised framework for Grid users, Grid resource providers and Grid application developers, by Kornmayer et al. introduces an eco-system to access Grid infrastructures with support for contextualised user roles. The abstraction layer of the g-Eclipse system, its integration in the Eclipse framework and the main use cases are presented.

Zuzek et al. propose a model to support the process of setting up a Virtual Organization (VO) in their paper Formal Model for Contract Negotiation in Knowledge-based Virtual Organizations. They discuss in detail the formal model underlying the process of contract negotiation and how the ontological description of domains related to given VO supports this process.

In An Approach for Enriching Information for Supporting Collaborative eWork, Anya et al. combine latent semantic analysis, domain task modelling and conceptual learning to enrich information in order to support collaborative e-Work. They illustrate their approach using the prototypical e-Workbench system.

The contribution by Dunk et al., Dynamic Virtual Environments Using Really Simple Syndication, investigates the use of really simple syndication (RSS) to dynamically change virtual environments (VE). Instead of simulating weather conditions in training scenarios, actual weather conditions can be incorporated, improving the scenario and immersion. This weather data is gathered by incorporating an up-to-date RSS feed in the VE.

Jamieson et al. present another contribution from the Virtual Reality domain. In Immersive Co-operative Psychological Virtual Environments (ICPVE) they discuss their approach to develop a variety of different applications from the field of psychology, using a single framework.

In the paper Environment for collaborative development and execution of virtual laboratory applications by Funika et al., a user interface system is introduced which enables collaboration between developers and users, to improve experiments and introduce more refinements to the research conducted within the ViroLab Virtual Laboratory. 